\title{
Review Article \\ Recent Advancements in Autonomous Robots and Their Technical Analysis
}

\author{
Boni Liu \\ School of Electronic Engineering, Xi'an Aeronautical University, Xi'an 710077, China \\ Correspondence should be addressed to Boni Liu; 200607004@xaau.edu.cn
}

Received 8 December 2020; Revised 7 January 2021; Accepted 19 January 2021; Published 3 February 2021

Academic Editor: Bhawani Shankar Chowdhry

Copyright (c) 2021 Boni Liu. This is an open access article distributed under the Creative Commons Attribution License, which permits unrestricted use, distribution, and reproduction in any medium, provided the original work is properly cited.

\begin{abstract}
The purpose of this paper is to discuss and present a technical analysis of the recent advancements in autonomous robots equipped with a manipulator. The autonomous robots include unmanned aerial vehicle (UAV), unmanned underwater vehicle (UUV), and unmanned ground vehicle (UGV). A manipulator can make an autonomous robot more adaptable and robust but it can also affect its performance as well. Several issues can arise because of the installation of a manipulator like the robot becoming unstable due to the extra weight, slow convergence, and errors in the path planning. Therefore, this study presents the numerous recent techniques that are in use to counter the aforementioned problems. The methodology and approach used in this paper are to first present the dynamic model of the autonomous robot. Then, the study offers a performance analysis of the specific robot in question. Finally, the paper formulates the limitations of the recently proposed techniques in the form of a table for each vehicle. The key findings of this study are a comprehensive review of the aforesaid techniques and their technical analysis. The unique contribution of this study is to present some of the limitations that these methods have so the researcher can better select the method according to the mission requirement.
\end{abstract}

\section{Introduction}

For the past few years, the researchers are engaged in evaluating the performance of autonomous vehicles with the addition of manipulator design due to the emerging demand in executing the number of flexible tasks in any dull, dirty, difficult, or dangerous environment [1-3]. These manipulators provide easy access to perform several jobs with merely small inertia, high load to weight ratio, and smart flexible structure [4]. For complex dynamic models with time delays in output variables and unmodeled dynamic factors, high-performance tracking has been observed as still one of the challenging tasks.

Acquiring the real and precise dynamics of the system during control design is among the complicated and strenuous activities of the procedure. The researchers in this regard are opting for some hybrid-type control algorithms to improve the tracking performance [5-7]. One may design such control designs, but they require the tuning of several parameters. In short, one researcher has two tough approaches either to acquire an exact mathematical dynamic model of autonomous vehicles or to estimate the numerous parameters for control design to produce refined input logic for the proposed system. Before going through the literature review, one should understand the types of autonomous unmanned vehicles. These vehicles are autonomous because of their ability to perform any sort of task without any intervention of human beings. Figure 1(a) shows a UUV with a manipulator [8], Figure 1(b) shows a UAV with a manipulator [9], and Figure 1(c) presents a UGV with a manipulator [10].

This entire review paper discusses the four types of unmanned vehicles embedded with gripper, that is, unmanned underwater vehicle (UUV), underactuated quadrotor unmanned aerial vehicle (QUAV), unmanned ground vehicle (UGV), and last but not least unmanned air-cushion vehicle (UACV). In addition to this, the paper addresses the constraints such as the occurrence of time delays and exogenous disturbances in a system.

The idea for embedding the UUV with a manipulator is introduced many times, that is, $[11,12]$. This is because of 


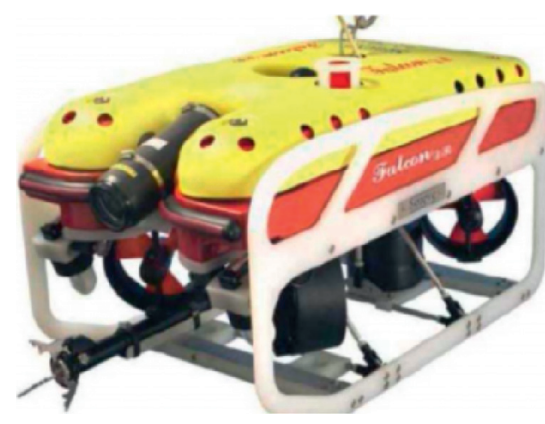

(a)

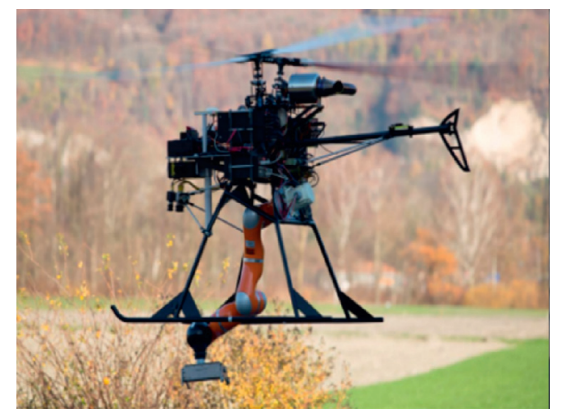

(b)

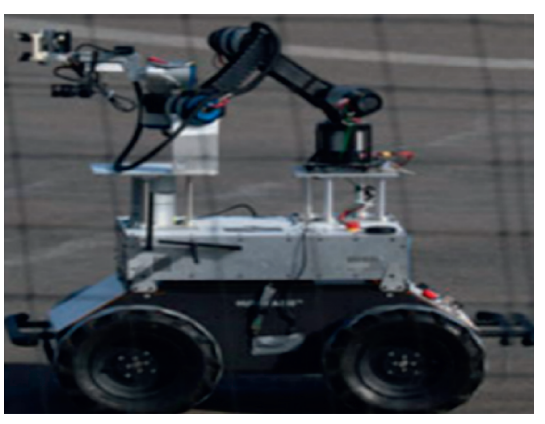

(c)

FIgURE 1: Types of unmanned vehicles embedded with a manipulator.

enabling an ability to grasp the target in water. Most of the UUVs have six degrees of freedom but only four actuators which make them underactuated system. These underactuated systems are very hard to control. Thus, for the stabilization, various hybrid control designs are introduced by researchers. In the catalog of such controllers, one may see model reference adaptive control (MRAC), sliding mode control (SMC), and many other robust control strategies [13-16].

Since the last decade, the extensive use of UAVs has been observed in various fields, either for commercial purposes, that is, surveillance $[17,18]$, or for military-oriented tasks. This type of unmanned vehicle got a great boom because of its aggressive maneuverability [19-22] over a long field of distance. Researchers have also tried to embed smart manipulator/gripper mechanism $[23,24]$, with UAV in order to increase the utility of drones in multiple fields. Researchers previously proposed commonly 01 and 02 DOF-based manipulators with unmanned aerial vehicles, that is, quadrotor [25]. Researchers were engaged initially in optimizing the control performance for the control law associated with the above manipulators [26]. The researchers also proposed some advanced mechanical designs and typical construction of quadrotor embedded with grippers of lightweight but with great capability to grasp the object within the working envelop [27].

Researchers also embedded some smart manipulators on such UGVs, that is, [28]. These smart manipulators have increased the manipulating ability to move up to $250 \mathrm{~kg}$ mass from one point to another. It is believed that a UGV must have good speed and navigation systems to monitor and manipulate the objects within harsh terrain [29]. Thus, in literature, one may find several types of manipulators as discussed by [30-32]. In most cases, it is recommended to use servo motors for ideal torque and mass ratio. In addition to this, a servo motor can be controlled easily. In today's era, researchers proposed different microcontrollers for experimental design, that is, Raspberry Pi [30], Arduino [33], or any modular programmable logical controller (PLC).

The motivation behind this paper was to collate the research studies about autonomous robots in one place so that new authors and researchers can easily compare the benefits and limitations of each study and pick the one most optimal for their mission requirement.
The main contributions of the paper are to provide one comprehensive review and technical analysis of the old and new studies about the UUVs, UAVs, and UGVs, to shed light on the limitations of the aforementioned studies in the form of an easily accessible table.

The paper is arranged as follows: Section 2 presents some cutting-edge research into autonomous robots. Section 3 discusses the UUV with the manipulator, its dynamic model, and its performance analysis and finally sums up the limitations of the previous techniques in a table. Similarly, Section 4 deals with the UAV, and Section 5 handles the UGV. Then, Section 6 provides technical analysis, and lastly, Section 7 concludes the whole study.

\section{State of the Art}

The state-of-the-art approach for UUV is discussed in [34] where a nonlinear observer-based model is amalgamated with dual proportional integral derivative (Dual-PID) design. This research provides comparatively effective results for 06 degrees of freedom (DOF) UUV with 02 DOF manipulator.

Researchers in [35] present a state-of-the-art technique for UAVs using $5 \mathrm{G}$ networks in a smart city. The researchers use blockchain-based solutions to secure these $5 \mathrm{G}$ networks for industrial and defense purposes.

Academics in [36] offer a novel idea of integrating UGV and UAV for construction site data collection. The UGV is autonomous and travels using the help of its sensors and the UAV which alerts it of any danger not visible to UGV on the ground.

\section{Unmanned Underwater Vehicle Equipped with Manipulator Design}

For repairing the structures, mostly in the offshore oil industry, these UUVs are highly recommended. This is because of their capability to reach in the depth of the sea unlike humans [37]. These UUVs have been blessed with two main abilities, that is, position stalking and dynamic stalking. This means that a UUV can maintain all positions throughout time with respect to the body.

One should not forget about the underwater dynamics that can lead to huge turbulences. These underwater 
dynamic factors are hydrodynamic coefficients and the mass flow rate through water inlets [11].

Discussing the previous works related to UUV, in [14], the author addressed the behavior by using SMC. An extended version, that is, higher-order SMC (HOSMC) can also be seen in [16] where the chattering phenomena (high number of oscillations) were reduced using higher order of SMC [13]. In some of the research works, one may see the use of a dual control scheme, that is, using proportional derivative (PD) and proportional integral control (PID) controller to stabilize the underactuated dynamics of UUV. Since this PID and PD, dual scheme produces fine results but in the presence of nonlinearities, this will never hold up the response for so long and shall lead it towards instability.

Researchers [13] proposed multivariable sliding mode control for the stabilization of attitude and position of a UUV equipped with a manipulator. In this case, the proposed UUV is a fully actuated system (number of control inputs are equal to degrees of freedom) that is why it can grasp any object underwater easily but simultaneously the power consumption by the actuators is huge as compared to underactuated UUVs.

3.1. Dynamic Model of UUV. The dynamic model for UUV is achieved after going through the study of both frames of references, that is, Earth frame of reference (inertial frame) and fixed body frame (noninertial frame). The common design is comprised of six actuators that lead to six DOF easily [38]. In this subsection, the main idea related to the state-of-the-art approach is discussed.

Here, the Dual-PID control techniques are fused with the nonlinear model-based observer to stabilize the fully actuated underwater vehicle. This strategy is applied on six DOFbased UUVs embedded with a gripper/manipulator of two DOF; this makes in total eight DOF to control. Figure 2 shows a six DOF UUV physical model embedded with a manipulator [39]. Table 1 presents the orientation, translational, and angular velocities, forces, and moments along with the degrees of freedom for the UUV.

The position and orientation with respect to the inertial frame are given as

$$
\begin{aligned}
& \mu_{1}(t)=\left[\begin{array}{l}
x \\
y \\
z
\end{array}\right], \\
& \mu_{2}(t)=\left[\begin{array}{l}
\varphi \\
\theta \\
\psi
\end{array}\right] .
\end{aligned}
$$

In equation (1), $\mu_{1}(t)$ and $\mu_{2}(t)$ are the vectors that describe the position and angular velocities of UUVs. The column matrix $\mu_{2}(t)$ is also known as the attitude of the vehicle.

$$
\begin{aligned}
& T_{1}(t)=\left[\begin{array}{lll}
u & v & w
\end{array}\right]^{T}, \\
& T_{2}(t)=\left[\begin{array}{lll}
p & q & r
\end{array}\right]^{T},
\end{aligned}
$$

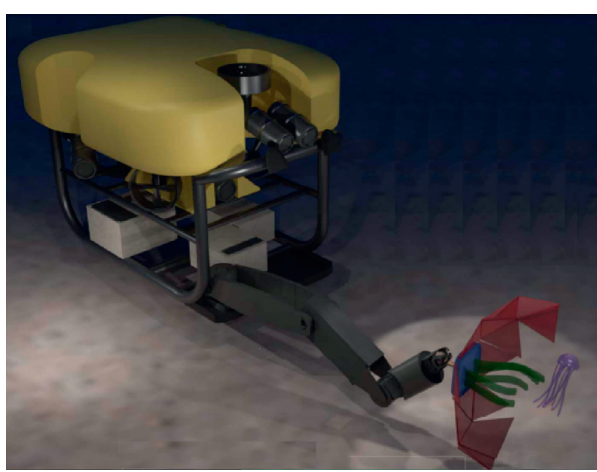

FIGURE 2: UUV with a manipulator.

where $T_{1}(t)$ represents the translational velocities whereas $T_{2}(t)$ represents the angular velocities. By combining the translational and angular velocities as in [38], we get

$$
v(t)=\left[T_{1}(t), T_{2}(t)\right]^{T} \varepsilon \Re^{6} .
$$

The rotation matrix can be derived using Newton-Euler methods as

$$
R=\left[\begin{array}{ccc}
c_{\theta} c_{\psi} & -c_{\theta} c_{\psi}+c_{\psi} s_{\phi} s_{\theta} & s_{\phi} s_{\psi}+c_{\phi} c_{\psi} s_{\theta} \\
c_{\theta} c_{\psi} & c_{\phi} c_{\psi}+s_{\phi} s_{\theta} s_{\psi} & -c_{\psi} s_{\phi}+c_{\phi} s_{\theta} s_{\psi} \\
-s_{\theta} & c_{\phi} c_{\theta} & c_{\phi} c_{\theta}
\end{array}\right],
$$

where $c_{x}$ means $\cos (x)$ and $s_{x}$ means $\sin (x)$. The dynamic model for UUV is shown as follows:

$$
\begin{aligned}
M(t) \dot{v}(t) & =g(v(t)), \mu(t), U(t)+\tau_{e(g)}, \\
\dot{\mu}(t) & =h(v(t), \mu(t)) .
\end{aligned}
$$

In the above set of equations, $M(t)$ is the inertial matrix that comprised hydrodynamic mass change and functions (e.g., one can see (.) and $h(.) \varepsilon \mathscr{R}^{6 x 1}$ ). $M(t)$ is the sum of centripetal mass and Coriolis body mass mentioned as follows [40]:

$$
M(t)=M_{C R}(t)+M_{C P}(t) .
$$

3.2. Kinematics of $U U V$. As per the conventional study by [41], the kinematic set of equations are given as in equations (7) and (8):

$$
\begin{aligned}
& \left\{\begin{array}{l}
\dot{\varphi}=p+q \sin \varphi \cdot \tan \theta+r \cos \tan \theta \\
\dot{\theta}=q \cos \varphi \cdot-r \sin \varphi \\
\dot{\psi}=\frac{q(\sin \varphi+r \cos \varphi)}{\cos \theta}
\end{array}\right. \\
& \left\{\begin{array}{l}
\dot{x}=u\left(c_{\theta} c \psi\right)+(s \theta s \varphi c \psi-s \psi c \varphi) v+(s \theta c \varphi s+s \psi s \varphi) \\
\dot{y}=u\left(c_{\theta} s \psi\right)+(s \theta s \varphi s \psi+c \psi c \varphi) v+(s \theta c \varphi s \psi-c \psi s \varphi) w \\
\dot{z}=-u s_{\theta}+(c \theta s \varphi) v+(c \theta c \varphi) w
\end{array}\right.
\end{aligned}
$$


TABLE 1: Symbols for the 6 DOF UUV.

\begin{tabular}{lccc}
\hline Position & Linear and angular velocities & Forces and moments & Degree of freedom \\
\hline$x$ & $u$ & $X$ & Surge \\
$Y$ & $v$ & $Y$ & Sway \\
$Z$ & $w$ & $Z$ & Heave \\
$\phi$ & $p$ & $K$ & Roll \\
$\theta$ & $q$ & $M$ & Pitch \\
$\psi$ & $r$ & $N$ & Yaw \\
\hline
\end{tabular}

3.3. Modeling of Manipulator Design. For modeling the manipulator design, one should consider the moments of the arm as an external torque. Since the attached manipulators connected with UUV are based on two links and one joint mostly using a simple servo motor. The kinematics for this gripper/manipulator is stated by [42] via opting for direct kinematics. This method helps to compute the orientation by finding the nth number of joints and compute the position of the end effector to grasp the object correctly.

Thus, the nth number of joints can be expressed as $q \in R^{n}$, whereas the position of the end effector is expressed as $\left\{n_{p} \in R^{3}, n_{0} \in R^{3}\right\}$. One can now develop a relationship between position and orientation easily as provided as follows:

$$
n_{p, o}=\left[n_{p}, n_{o}\right]^{T} \in R^{6}
$$

Researchers have used the Denavit-Hartenberg (DH) formulation to find the configuration of an end effector of the gripper. Moreover, the dynamics of UUV stated that the total forces and torque that are acting on the body of UUV in the deep sea can be expressed in generic as

$$
\left\{\begin{array}{l}
f_{i}=m_{i}\left[a_{i}+\dot{T}_{2}(t) \times r_{i}+T_{2}(t) \times\left(T_{2}(t) \times r_{i}\right)\right], \\
T_{m}=I \times \dot{T}_{2}(t)+T_{2}(t) \times\left(I \times \dot{T}_{2}(t)\right] .
\end{array}\right.
$$

In equation (10), $m_{i}$ is an additional mass due to the manipulator and the weight of the object that must be grasped by the manipulator. Moreover, $r_{i}$ is the vectoral distance from the origin frame $I$ towards the center of gravity of the link. The variable $a_{i}$ is the translational acceleration from the origin of the frame, whereas $\dot{T}_{2}(t)$ is the vector denoting the change in angular velocities where $T_{2}(t)$ is the vector consisting of rotational velocities.

\subsection{Performance Analysis of UUV Equipped with} Manipulator. There is an effective need for an autonomous unmanned underwater vehicle due to several issues. The important thing at this moment is to save the lives of our divers and get efficient results by using UUVs beneath the sea more than the depth covered by divers. Figure 3 shows a UUV equipped with a manipulator that has multiple links and joints to grasp the object [38].

Researchers also used the bioinspired dolphin algorithm for controlling the locomotion of UUVs like a real dolphin. Figure 4 presents a UUV hardware design based on a bioinspired dolphin algorithm [11].

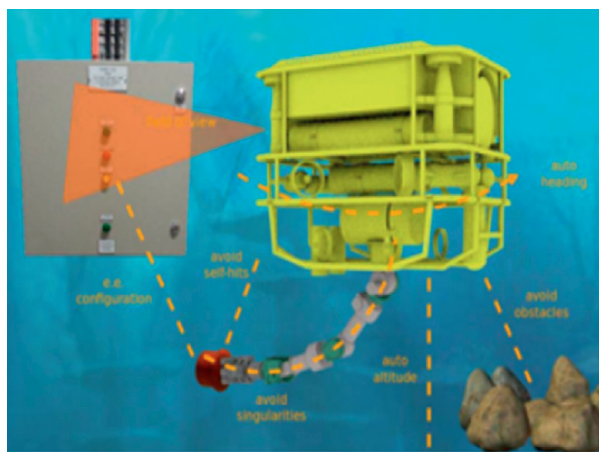

FIGURE 3: UUV equipped with a manipulator.

Using the Newton-Euler dynamic method shown in Figure 5, we can derive the equation of motions [40]. This method is easy but has some limitations such as gimbal lock due to singularity issues.

After going through the latest papers and current stateof-the-art approaches, Table 2 presents the previously proposed techniques for UUVs, the hardware they are applied on, and their limitations.

\section{Underactuated Quadrotor UAV Equipped with Manipulator Design}

Like underwater vehicles, unmanned aerial vehicle and its dynamic model are also derived from the Newton-Euler method. This method is frequently opted for by various researchers because of less complexity. The only limitation of this approach is the gimbal lock due to the singularity issue which can be reduced, not eliminated completely through hyperbolic tangent function. These equations involve the trigonometric functions; hence, the computation time for these terms is usually huge, and therefore expensive programmable controller is selected which leads to an expensive hardware design [44].

The focus for the UAVs in this paper is set on quadrotor type of UAVs. This is because of a fewer number of actuators that result in less power consumption and long battery time for flight [44-46]. Researchers modified the quadrotor with multirotors as well as the manipulator designs too. There are also some hybrid control schemes too, previously proposed by $[47,48]$, for the stabilization of the entire behavior of UAV with a gripper mechanism. The same control laws that were proposed before for UAV are proposed here too such as model reference adaptive control, a hybridized version with sliding mode control for quadrotor UAV (QUAV) equipped with 2 DOF manipulator [49]. 


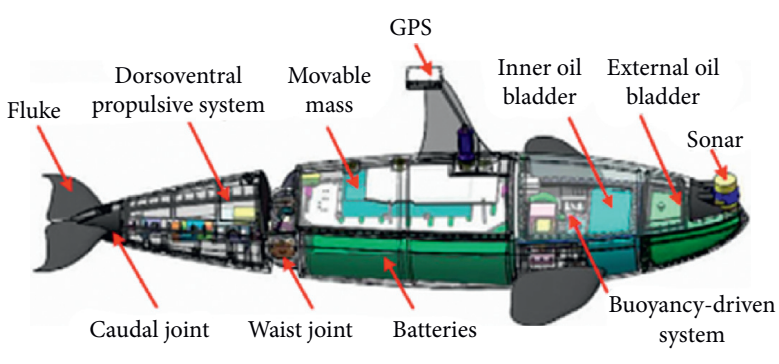

(a)

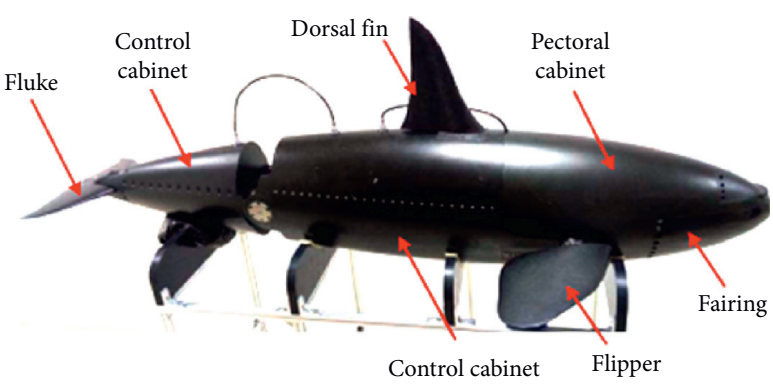

(b)

Figure 4: UUV hardware design.

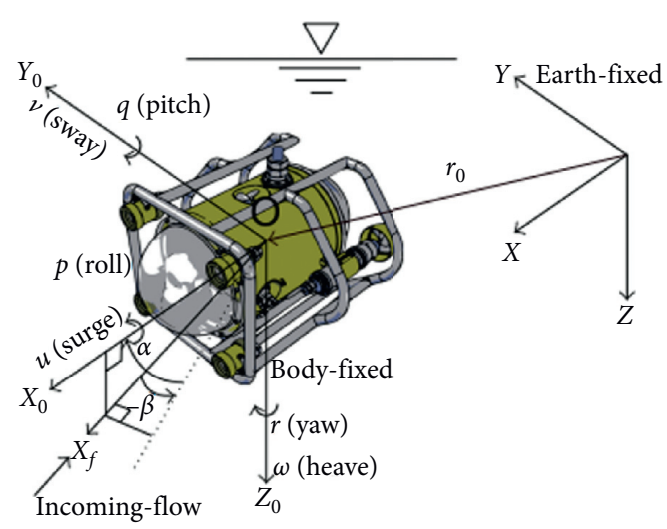

Figure 5: Deriving the equations of motion using Newton-Euler method.

4.1. Performance Analysis of UAV Equipped with Manipulator. The paper discusses two categories of unmanned aerial vehicles. One is a cable-driven UAV and the second one is equipped with a manipulator design. The current approaches for the trajectory tracking of UAVs are discussed by $[50,51]$, in which an adaptive robust control law is proposed. The lumped dynamics are estimated using estimator design and in addition to this, the chattering phenomenon is also eliminated [52]. For unknown modeled factors, one can see several such hybrid techniques. Researchers have also proposed subcontrol blocks, that is, time delay estimator, supertwisting law, and fractional-order SMC technique to minimize the dynamic error in the system. Researchers in this way obtained $20 \%$ efficiency for quadrotor equipped with 2 DOF manipulator on a referred path [53]. The hybridized version of the regulation, pole-placement, and tracking (RST) control design with MRAC and the stability for tracking and grabbing the objects both are proved using MIT rules. The work proposed by [53], where quadrotor varies its mass from $0.5 \mathrm{~kg}$ to $5.0 \mathrm{~kg}$, and the manipulation related parameters are achieved using the Denavit-Hartenberg principle. Table 3 presents the previously proposed techniques for quadrotor UAVs, the hardware they are applied on, and their limitations.

4.2. Dynamic Model of QUAV with 2 DOF Gripper. Researchers avoided the different parameters, that is, aerodynamic effect, ground effects, and flapping of blades, and proposed an overall dynamic model. Figure 6 shows the overall model of a dynamic quadrotor with a 2 DOF manipulator $[53,54]$. The Newton-Euler method is the most frequent method used and stated by the majority of the people. One can find the separate models as well like in [54] but this will increase the complexity.

The overall mathematical model including the gripper dynamics is derived and stated as

$$
\left[\begin{array}{c}
f_{q}(U) \\
T_{q}\left(U, q_{A}^{j}\right)
\end{array}\right]+\left[\begin{array}{c}
f_{A}\left(\partial q_{A}^{j}\right) \\
T_{A}\left(\partial q_{A}^{j}\right)
\end{array}\right]=\left[\begin{array}{cc}
m \bar{I} & 0 \\
0 & I\left(q_{A}^{j}\right)
\end{array}\right]\left[\begin{array}{c}
\dot{v} \\
\dot{\omega}
\end{array}\right]+\left[\begin{array}{c}
0 \\
\omega \times I \omega
\end{array}\right] .
$$

In equation (11), the left-hand side is the dynamics of quadrotor and manipulator, respectively, whereas the righthand side is the vector form of rigid quadrotor body. The term like $f_{q}(U)$ is the control input force and torque given as $T_{q}\left(U, q_{A}^{j}\right)$ for quadrotor whereas the force exerted by manipulator is given as $f_{A}\left(\partial q_{A}^{j}\right)$ and torque as $T_{A}\left(\partial q_{A}^{j}\right) . m$ is the total mass and $I$ is an identity matrix of $(3 \times 3)$ order. $I\left(q_{A}^{j}\right)$ is the total inertia. Moreover, $\dot{v}$ and $\dot{\omega}$ are angular velocities.

\section{Unmanned Ground Vehicle Equipped with Manipulator Design}

5.1. Dynamic Model of UGV with Manipulator Designs. As discussed, these unmanned vehicles are deployed in such tasks that are far away from human management. UGVs are among the prominent vehicles that are used for surveillance purposes and can tackle high-risk crises [28]. The part of deploying sensors is the core part that guides the UGV in indoor/outdoor space.

The main motive of proposing the UGV equipped with manipulator design is to navigate it in a space with a specific trajectory tracking and manipulating the objects to mentioned coordinates. Generally, two approaches are commonly adapted with control designs such as mentioned as follows: understanding of failure modes [55].

5.2. Failure Analysis of Acquired Data and Its Usage. The researchers opted for the Newton-Euler method most frequently for UGVs like UUVs and UAVs. Here, the robotic manipulator and the payload duly manipulated are driven using a free diagram as shown in Figure 7 [56] 
TABLE 2: Limitations of previously proposed techniques for UUVs.

Techniques (PID) nonsingular fast terminal sliding mode control (STF-PID-NFTSM)

Nonlinear disturbance observer-based sliding mode control law

Bioinspired dolphin algorithm embedded with disturbance rejection scheme

Adaptive iterative approach with boundary layer and hyperbolic tangent function

Underwater long-arm manipulator (ULAM) with an improved hydraulic driving system (SHDS) with fuzzy-based PID control

The nonlinear model-based observer design using the linearization of the model to estimate the current state

DexROV, an EC Horizon 2020-funded project

Hybrid high-order terminal sliding mode (HHOTSM) control approach for (MIMO) uncertain nonlinear systems

Sliding mode control-based dynamic positioning system

Applied on ship model in the deep sea

UUV with TITAN4 from shilling robotics

UUV with long-arm gripper/manipulator

Underwater robotic manipulator

Flexible joint-based

Supervisory feedforward artificial neura network- (ANN-) based fuzzy control law to address friction and elasticity issues of a manipulator

manipulator design

PID guidance and control laws to perform basic control tasks such as autoheading, autospeed, and straight line

Unmanned surface

vehicle
UUV Visor3

Limitations

References

(1) The average convergence time is 3.5 seconds in

the presence of uncertainties

(2) The response experiences an undershoot in the presence of unmodeled uncertainties

(1) The proposed UUV is a fully actuated system and thus consumes huge power and process time

(2) It has a small working envelope and does not consider unmodeled dynamic factors

(3) For estimating the sudden hydrodynamic coefficients, the strategy is complex

(1) The disturbance taken in the simulation is limited up to $0.01 \sin (t)$

(2) Undersea there are so many other factors, that is, hydrodynamic coefficients which were assumed through ANSYS and computational fluid dynamics

(1) Bounded conditions have been defined already

(2) Control input somehow experiences the chattering-like noise

(1) Either in low or in high pressure, there is an average tracking error of 5.12 percent

(2) Each joint experiences an average overshoot of $1.5 \%$ and a steady-state error of 0.015

(3) Fuzzy-based PID slows down the maneuverability and increases hardware cost (1) Chattering effect is available along with some transient and steady-state issues

(2) Hardware implementation will be costly as compared to other previously proposed UUVs

(1) The output response, that is, angular and

translational velocities, experiences a chattering

effect too with reasonable tracking error because of a long length of gripper and time delays

(1) First convergence time but output has a response which has some overshoots too

(2) Chattering is still there due to switching mode $\mathrm{b} / \mathrm{w}$ terminal SMC and higher-order SMC

(1) parameters were assumed for fully loaded and ballasted conditions and did not consider unmodeled dynamic factors

(2) Responses, that is, surge, sway, and yaw, experience $12 \%-20 \%$ overshoots in simulations and experimental work, respectively

(1) There is a reasonable error in load and the motor trajectories

(2) The convergence rate on the time axis is not suitable for any sensitive pick and drop tasks

(3) The technique is based on ANN and a fuzzy set of rules; it will only be implemented on fieldprogrammable gate array (FPGA) or digital signal processing (DSP) kits that lead us to an expensive hardware design

(1) Time delays due to Global Positioning System(GPS-) based communication.

(2) Due to these time delays, the responses have tracking errors 
TABLE 3: Limitations of previously proposed techniques for Quadrotor Unmanned Aerial Vehicles.

$\begin{array}{llll}\text { Technique } & \text { Applied on } & \text { Limitations } & \text { References }\end{array}$

Adaptive control-based regulation, poleplacement, and tracking (RST) control law

Adaptive time delay control (ATDC) scheme using a fractional-order nonsingular terminal sliding mode (FONTSM)

Continuous nonsingular fast terminal sliding mode (CNFTSM) control scheme using a modified supertwisting algorithm (STA)

A composite controller scheme using 02 subcontrol blocks (one for arm/gripper and second for QUAV), gain tuning method

An adaptive terminal sliding mode controller for the trajectory tracking of robotic manipulators using radial basis function neural networks (RBFNNs)

Combination of gain scheduling and Lyapunovbased model reference adaptive control (MRAC)

Backstepping control design using an admittance subcontrol block for the manipulator design

Proportional integral derivative- (PID-) based wireless control of quadrotor at hovering state

Nested controller scheme for attitude stabilization, vision-based navigation, and guidance, with the aerial gripping

Direct approximate-adaptive control using cerebellar model arithmetic computer (CMAC) nonlinear approximators

Discrete proportional integral derivative control QUAV with $4 \mathrm{~g}$ weight design

QUAV with 2 DOF
gripper

Cable-driven

QUAV with a cabledriven manipulator

QUAV with a cabledriven manipulator

Robotic manipulators

UAV for manipulation

Quadrotor with multiple payload variation and $1 \mathrm{~kg}$ payload mass manipulators

(1) Sudden fluctuations are experienced due to

variation in altitude

(2) Sluggish in grasping the objects (need an improvement for fast grasp)

(1) The tuning function for ATDC is a complex procedure

(2) The root means square error for joint $01 / 02$ is 0.3 and 0.32 .5 chattering appears at torque responses in the presences of payload

(1) The response experiences chattering in experimental work

(2) The software simulations also show some overshoots too

(3) There is also a steady-state error available for manipulator

(1) Gimbal lock due to Newton-Euler method

(2) Due to cosine and sine terms, the computation time increases; therefore, an expensive DSP kit is proposed for the algorithm

(3) The chattering phenomenon appears while real flight takes off and path planning

(1) In the presence of any disturbance factor, that is, wind disturbance, the robotic manipulator will be diverted from trajectory for some time and refollow the trajectory again

(1) There is undershoot and overshoot in yaw angular velocity

(2) While performing pitch, the rotors experience oscillations for 10 seconds

(1) Due to the change of the reference values, too

Octa-copter UAV with fast several joints and their servos are not able to 07 DOF manipulator follow, and the response lags the reference for some time

(1) The major shortcoming is the control using the Zigbee module which is sluggish and hence

Quadrotor with a QUAV will never be able to perform aggressive payload of 250 grams maneuvers

(2) At the payload of 250 grams, the QUAV cannot balance itself in a hovering state

(1) There is an error in both hovering and path tracking states (i.e., the deviation in between $\pm 13 \mathrm{~cm}$ ). At outdoor operations, this error increases up to $\pm 20 \mathrm{~cm}$.

(2) During wind disturbance, the quadrotor deviates from the path for a few seconds and refollows the path again.

(1) There must not be extreme variation in payload mass, and this will lead to bursting or instability because of the previously trained weights

(1) After visualizing the pitch and roll response, there are drastic oscillations in the output response due to the ground effect 


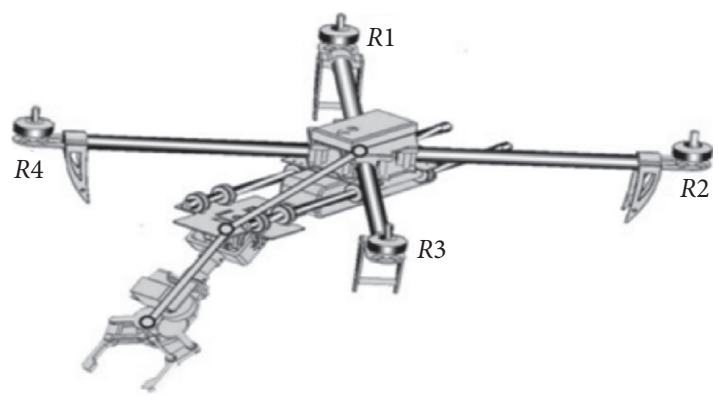

FIgURE 6: UAV with a manipulator.

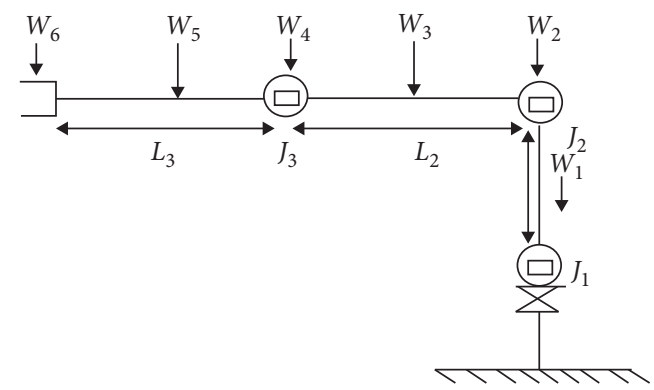

FIgURE 7: Manipulator-free diagram for torque.

$$
T=F L,
$$

where " $T$ " is the torque, " $F$ " is the force in Newton, and " $L$ " is the perpendicular distance between the point of rotation and applied force.

$$
F=W=m g .
$$

The term " $m$ " is the mass " $g$ " which is the gravitation acceleration; hence, with equation (13), equation (12) can be transformed as

$$
T=m g L
$$

Researchers have proposed various dynamic models for UGV and its manipulators such as in [57]. Figure 8 presents an example of a UGV [31] and a manipulator design [56].

Table 4 presents the previously proposed techniques for UGVs, the hardware they are applied on, and their limitations. Moreover, Table 5 summarizes Tables 2-4 into one easily comprehensible table to further elucidate the shortcomings of all the techniques used for an unmanned vehicle.

\section{Technical Analysis and Discussion}

If someone is working on UUV, then one should work on the constraints, that is, chattering effect, producing cost-effective hardware design, minimizing the power consumption, and process time. This manuscript provides an opportunity to evaluate either robust or adaptive control laws with nonlinear observer designs.

Table 2 presents the previously proposed techniques for UUVs, the hardware they are applied on, and their limitations. It is a helpful guide for any future researchers to

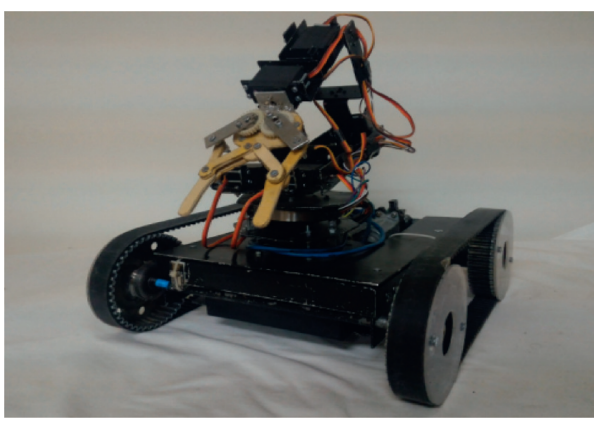

(a)

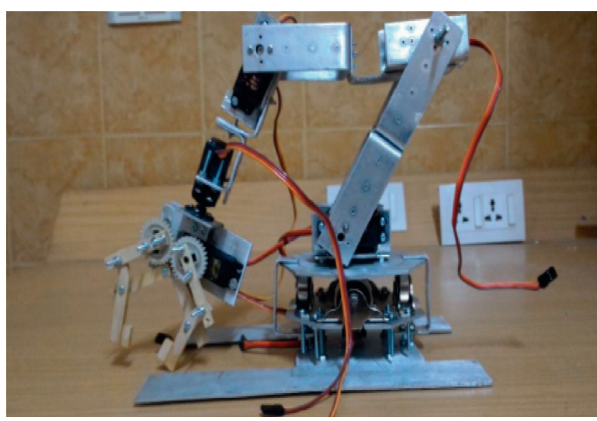

(b)

FIgURE 8: UGV with a manipulator. (a) UGV model. (b) Manipulator design.

choose the best strategy according to their mission requirements.

For quadrotor UAV, one must concern the issues such as the elimination of gimbal lock, chattering noise, and some serious undershoots/overshoots due to unmodeled dynamic factors. The paper suggests a serious need for reviewing the adaptive control law and their amalgamation with state observer design. The emerging bioinspired algorithms such as the pigeon algorithm are recommended while designing the observer design.

Table 3 presents the previously proposed techniques for quadrotor UAVs, the hardware they are applied on, and their limitations. Any future academic researching this field would find this table useful for deciding the best technique for their study.

For UGV, the processing time and hardware designs are emerging issues, and hence paper reviewed some of the fuzzy logic-oriented designs which produce fine response outcomes but are slower due to the fuzzy inference system. Therefore, it is suggested to use a single dimension-based fuzzy logic controller as they minimize the processing time. Once the processing time will be reduced, then a hardware designer may opt for a cheap microcontroller for programming.

Table 4 presents the previously proposed techniques for UGVs, the hardware they are applied on, and their limitations. It delineates the drawbacks of the mentioned strategies and would help in selecting the best method for the UGV.

After going through several research papers, Table 5 has been stated in this paper. This table shows the limitations of 
TABLE 4: Limitations of previously proposed techniques for Unmanned Ground Vehicles.

\begin{tabular}{llll}
\hline Technique & Applied on & Limitations
\end{tabular}

(1) NRF module is used to communicate with UGV and hence with the displacement in the antenna's direction may lead to loss of connection.

(2) With 06 DOF, still there are certain easy moves that it cannot do and with the UGV prototype, it consumes relatively high power.

Computer vision and control approach

GV with variable payload up to $4 \mathrm{~kg}$ and 6 DOF manipulator

Computer vision and Internet of things (IoT) control approach

Novel hybrid additive-subtractive manufacturing (HASM)

DTMF-based control law for robotic arm

European ICARUS project towards the development of unmanned search and rescue (SAR) robots

An alternative and comprehensive mapgenerating algorithm

The design and control of robotic search and rescue system based on an immunocontrol framework

CoMoRAT (configurable mobile robot for all-terrain applications) for the installation of variable payloads clear sewerage pipelines

06 DOF robotic manipulator tree climber

UGV with navigation systems

Modular UGV
(3) Without load, its RPMs are between 50 and 70.

The approach may not be affordable for heavy payloads. The image processing approach is restricted to only color. It is studied that various approaches recommend tracking object with respect to color, shape, and histogram

UGV with sharp rudder to

(1) The response and work are fine but the time delay is experienced in the response due to the internet of things and the sensor's resolution factor

(1) Due to fast response, there is a time delay in system response

(2) Sudden overshoots are also experienced

(1) The simple dual tone multiple frequency-based control is proposed which may face serious distortions in the presence of any external noise.

Robotic arm for a coconut (2) The approach is involved in cutting and climbing on coconut trees to pick and drop the coconuts. Thus, the minor error may destabilize the system and the cutter part may harm any human standing beneath (hazardous in nature).

(1) Focused on only rescue missions but there are

UGV with large gripper some huge tracking errors while performing manipulation of the objects

(1) The trajectories are fine with no error but in the absence of unmodeled factors

UGV with 2 DOF gripper

2 The output response for gripper experiences the steady-state error of $0.55 \%$ (1) The communication is performed using a Zigbee module, which is sluggish and thus brings time delay in responses

(1) The modular approach is presented that leads to the installation of additional components on UGV to make it affordable for variable load conditions (2) Expensive hardware approach

TABLE 5: Summary of all previously proposed control laws for all unmanned vehicles.

\begin{tabular}{|c|c|c|c|c|c|c|c|c|c|c|}
\hline \multirow{2}{*}{ References } & \multicolumn{9}{|c|}{ Limitations/shortcomings } & \multirow{2}{*}{ Vehicle type } \\
\hline & $\mathrm{TD}$ & OS/US & SC & $\mathrm{CE} / \mathrm{EN}$ & GL & SSE & EHD & PC & T_proc & \\
\hline [7] & & $\checkmark$ & $\checkmark$ & & $\checkmark$ & & & & & \\
\hline [39] & & & & & & & & $\checkmark$ & $\checkmark$ & \\
\hline$[11]$ & & $\checkmark$ & & $\checkmark$ & $\checkmark$ & & & & & \\
\hline$[6]$ & & & & & & & & & & \\
\hline$[43]$ & & & & & & $\checkmark$ & $\checkmark$ & $\checkmark$ & $\checkmark$ & \\
\hline$[40]$ & & & & $\checkmark$ & & & $\checkmark$ & $\checkmark$ & $\checkmark$ & UUVs with manipulators \\
\hline$[38]$ & & $\checkmark$ & & $\checkmark$ & & & & & & \\
\hline [16] & & & $\checkmark$ & & & & $\checkmark$ & $\checkmark$ & $\checkmark$ & \\
\hline$[14]$ & & & & $\checkmark$ & $\checkmark$ & & & & & \\
\hline [5] & & & $\checkmark$ & & & & $\checkmark$ & & & \\
\hline [13] & $\checkmark$ & & & & $\checkmark$ & $\checkmark$ & & & & \\
\hline
\end{tabular}


TABLE 5: Continued.

\begin{tabular}{|c|c|c|c|c|c|c|c|c|c|c|}
\hline \multirow{2}{*}{ References } & \multicolumn{9}{|c|}{ Limitations/shortcomings } & \multirow{2}{*}{ Vehicle type } \\
\hline & $\mathrm{TD}$ & OS/US & SC & $\mathrm{CE} / \mathrm{EN}$ & GL & SSE & EHD & PC & T_proc & \\
\hline$[53]$ & & $\checkmark$ & & $\checkmark$ & & & & & & \\
\hline$[50]$ & & & & & & $\checkmark$ & & & $\checkmark$ & \\
\hline$[52]$ & & $\checkmark$ & & & & & & & & \\
\hline [48] & & & & $\sqrt{ }$ & $\checkmark$ & & $\checkmark$ & $\checkmark$ & $\sqrt{ }$ & \\
\hline [49] & & & $\checkmark$ & & & & & & & \\
\hline [47] & & $\checkmark$ & & & & & & & & QUAVs with manipulator design \\
\hline [26] & $\checkmark$ & $\checkmark$ & & & & $\checkmark$ & & & $\sqrt{ }$ & \\
\hline [46] & $\checkmark$ & $\checkmark$ & & $\checkmark$ & $\checkmark$ & & & & & \\
\hline$[27]$ & & & $\checkmark$ & & $\checkmark$ & $\checkmark$ & & & & \\
\hline [45] & & & & & & & & & $\sqrt{ }$ & \\
\hline [44] & & & $\checkmark$ & $\checkmark$ & & & & & & \\
\hline [56] & $\checkmark$ & & & & & & $\checkmark$ & $\checkmark$ & $\checkmark$ & \\
\hline [58] & & & $\checkmark$ & & $\checkmark$ & $\checkmark$ & & & & \\
\hline [29] & & $\checkmark$ & & & & & & & & \\
\hline [59] & $\checkmark$ & & & & & & & $\checkmark$ & & UGV with maninulator decion \\
\hline [28] & & $\checkmark$ & & $\checkmark$ & & $\checkmark$ & & & $\checkmark$ & UGV with manipulator design \\
\hline [31] & & & & & $\checkmark$ & & & & & \\
\hline [30] & $\checkmark$ & & $\checkmark$ & & & & & & $\checkmark$ & \\
\hline [33] & & & & & & & $\checkmark$ & $\checkmark$ & $\checkmark$ & \\
\hline
\end{tabular}

TD: time delay. SC: slow convergence rate. GL: gimbal lock. EHD: expensive hardware. T_proc: process time. OS/US: over/undershoots. CE/EN: chattering effect/external noise effect. SSE: steady-state error. PC: power consumption.

all previously proposed control laws over the unmanned vehicles embedded with several manipulator types.

\section{Conclusion}

This review paper presents a detailed review of the current state-of-the-art approaches and control laws proposed already for three types of unmanned vehicles, that is, UUVs, UAVs (more specifically quadrotors), and UGVs. The manuscript comes up with the limitations in Table 5. By reading Tables 2 to 5 , one may see the most frequent problems in such unmanned vehicles, especially when embedded with manipulator design. The control laws so far proposed are fine until the degree of freedom for the manipulator is 02 .

If the DOF value increases, the tracking performance also degrades and one may experience the chattering noise and deviation from tracking for some time and refollow the path. In addition to this, there is also degradation in transient and steady-state performances, that is, steady-state error and slow convergence. These vehicles are designed for fast maneuvers and aggressive operations with greater reliability but with these constraints, these unmanned vehicles compromise on their overall performance.

For future research ideas, one could revisit the robust and adaptive control laws with an amalgamation of bioinspired algorithms and smart observer designs to manage these problems. Also, the current team is planning to evaluate the bioinspired algorithms for individual unmanned vehicle types and may come up with another review.

\section{Data Availability}

The data used to support the findings of this study are included within the article.

\section{Conflicts of Interest}

The author declares no conflicts of interest.

\section{Acknowledgments}

This paper was supported by the Science Research Fund of Xi'an Aeronautics University (Grant no. 2019KY0208).

\section{References}

[1] N. U. Rehman, K. Kumar, and G. E. M. Abro, "Implementation of an autonomous path planning \& obstacle avoidance UGV using SLAM," in Proceedings of the 2018 International Conference on Engineering and Emerging Technologies, ICEET 2018, pp. 1-5, Lahore, Pakistan, January 2018.

[2] J. Sánchez-García, "A survey on unmanned aerial and aquatic vehicle multi-hop networks: wireless communications, evaluation tools and applications," Computer Communications, vol. 119, pp. 43-65, 2018.

[3] G. E. M. Abro, B. Jabeen, and A. Manan, "Stabilization of nonholonomic 03 dof hovercraft using robust RST control design," in Proceedings of the Sukkur IBA Journal of Emerging Technologies, vol. 2, no. 1, pp. 45-50, Sukkur, Pakistan, August 2019.

[4] Y. Wang, S. Jiang, B. Chen, and H. Wu, "A new continuous fractional-order nonsingular terminal sliding mode control for cable-driven manipulators," Advances in Engineering Software, vol. 119, no. January, pp. 21-29, 2018.

[5] H. Chaoui, P. Sicard, and W. Gueaieb, "ANN-based adaptive control of robotic manipulators with friction and joint elasticity," IEEE Transactions on Industrial Electronics, vol. 56, no. 8, pp. 3174-3187, 2009.

[6] J. Wei, "Adaptive iterative learning control of a class of nonlinear time-delay systems with unknown backlash-like 
hysteresis input and control direction," ISA Transactions, vol. 70, pp. 79-92, 2017.

[7] M. Van, X. P. Do, and M. Mavrovouniotis, "Self-tuning fuzzy PID-nonsingular fast terminal sliding mode control for robust fault tolerant control of robot manipulators," ISA Transactions, vol. 96, pp. 60-68, 2020.

[8] C. Shen, B. Buckham, and Y. Shi, "Modified C/GMRES algorithm for fast nonlinear model predictive tracking control of AUVs," IEEE Transactions on Control Systems Technology, vol. 25, no. 5, pp. 1896-1904, 2016.

[9] M. J. Kim, K. Kondak, and C. Ott, "A stabilizing controller for regulation of UAV with manipulator," IEEE Robotics and Automation Letters, vol. 3, no. 3, pp. 1719-1726, 2018.

[10] J. Carius, M. Wermelinger, B. Rajasekaran, K. Holtmann, and M. Hutter, "Deployment of an autonomous mobile manipulator at MBZIRC," Journal of Field Robotics, vol. 35, no. 8, pp. 1342-1357, 2018.

[11] Z. Wu, J. Yu, J. Yuan, and M. Tan, "Towards a gliding robotic dolphin: design, modeling, and experiments," IEEE/ASME Transactions on Mechatronics, vol. 24, no. 1, pp. 260-270, 2019.

[12] C. Laschi, B. Mazzolai, and M. Cianchetti, "Soft robotics: technologies and systems pushing the boundaries of robot abilities," Science Robotics, vol. 1, no. 1, 2016.

[13] M. Caccia, M. Bibuli, R. Bono, and G. Bruzzone, "Basic navigation, guidance and control of an unmanned surface vehicle," Autonomous Robots, vol. 25, no. 4, pp. 349-365, 2008.

[14] E. A. Tannuri, "Dynamic positioning systems: an experimental analysis of sliding mode control," Control Engineering Practice, vol. 18, no. 10, pp. 1121-1132, 2010.

[15] F. A. Azis, M. S. M. Aras, M. Z. A. Rashid, M. N. Othman, and S. S. Abdullah, "Problem identification for underwater remotely operated vehicle (rov): a case study," Procedia Engineering, vol. 41, pp. 554-560, 2012.

[16] X.-T. Tran and H.-J. Kang, "Adaptive hybrid High-Order terminal sliding mode control of MIMO uncertain nonlinear systems and its application to robot manipulators," International Journal of Precision Engineering and Manufacturing, vol. 16, no. 2, pp. 255-266, 2015.

[17] J. Lee, D. H. Shim, S. Cho et al., "A mission management system for complex aerial logistics by multiple unmanned aerial vehicles in MBZIRC 2017," Journal of Field Robotics, vol. 36, no. 5, pp. 919-939, 2019.

[18] L. Li, Y. Zhang, M. Ripperger, J. Nicho, M. Veeraraghavan, and A. Fumagalli, "Autonomous object pick-and-sort procedure for industrial robotics application," International Journal of Semantic Computing, vol. 13, no. 2, pp. 161-183, 2019.

[19] N. Ammour, H. Alhichri, Y. Bazi, B. Benjdira, N. Alajlan, and M. Zuair, "Deep learning approach for car detection in UAV imagery," Remote Sensing, vol. 9, no. 4, p. 312, 2017.

[20] M. Hassanalian and A. Abdelkefi, "Classifications, applications, and design challenges of drones: a review," Progress in Aerospace Sciences, vol. 91, pp. 99-131, 2017.

[21] M. Silvagni, "Multipurpose UAV for search and rescue operations in mountain avalanche events," Geomatics, Natural Hazards and Risk, vol. 8, no. 1, pp. 18-33, 2017.

[22] G. Ding, Q. Wu, L. Zhang, Y. Lin, T. A. Tsiftsis, and Y.-D. Yao, "An amateur drone surveillance system based on the cognitive internet of things," IEEE Communications Magazine, vol. 56, no. 1, pp. 29-35, 2018.

[23] H. Bonyan Khamseh, F. Janabi-Sharifi, and A. Abdessameud, "Aerial manipulation-a literature survey," Robotics and Autonomous Systems, vol. 107, pp. 221-235, 2018.
[24] X. Ding, P. Guo, K. Xu, and Y. Yu, “A review of aerial manipulation of small-scale rotorcraft unmanned robotic systems," Chinese Journal of Aeronautics, vol. 32, no. 1, pp. 200-214, 2019.

[25] B. W. Davis, J. T. Diep, and S. Jose, (12) United States Patent, Moderna, vol. 2, no. 12, Cambridge, MA, USA, 2017.

[26] G. Heredia, "Control of a multirotor outdoor aerial manipulator," in Proceedings of the IEEE International Conference on Intelligent Robots and Systems, pp. 3417-3422, Chicago, IL, USA, September 2014.

[27] V. Ghadiok, J. Goldin, and W. Ren, "On the design and development of attitude stabilization, vision-based navigation, and aerial gripping for a low-cost quadrotor," Autonomous Robots, vol. 33, no. 1-2, pp. 41-68, 2012.

[28] D. Doroftei, A. Matos, and G. de Cubber, "Designing search and rescue robots towards realistic user requirements," Applied Mechanics and Materials, vol. 658, pp. 612-617, 2014.

[29] L. Li, A. Haghighi, and Y. Yang, "A novel 6-axis hybrid additive-subtractive manufacturing process: design and case studies," Journal of Manufacturing Processes, vol. 33, no. March, pp. 150-160, 2018.

[30] A. W. Y. Ko and H. Y. K. Lau, "Intelligent robot-assisted humanitarian search and rescue system," International Journal of Advanced Robotic Systems, vol. 6, no. 2, pp. 12-128, 2009.

[31] J. Suthakorn, "On the design and development of a rough terrain robot for rescue missions," in Proceedings of the 2008 IEEE International Conference on Robotics and Biomimetics, ROBIO 2008, pp. 1830-1835, Chicago, IL, USA, September 2001.

[32] J. M. Ed and D. Hutchison, "Autonomous vehicles," Auto Tech Review, vol. 64, 2012.

[33] G. Bayar, A. B. Koku, and E. I. Konukseven, "CoMoRAT: a configurable all terrain mobile robot," in Proceedings of the 11th Wseas International Conference on Automatic Control, Modelling and Simulation, pp. 507-512, Istanbul, Turkey, October 2009.

[34] Z. A. Ali, X. Li, and M. A. Tanveer, "Controlling and stabilizing the position of remotely operated underwater vehicle equipped with a gripper," Wireless Personal Communications, vol. 116, 2019.

[35] P. Mehta, R. Gupta, and S. Tanwar, "Blockchain envisioned UAV networks: challenges, solutions, and comparisons," Computer Communications, vol. 151, pp. 518-538, 2020.

[36] K. Asadi, A. Kalkunte Suresh, A. Ender et al., "An integrated UGV-UAV system for construction site data collection," Automation in Construction, vol. 112, p. 103068, 2020.

[37] P. I. Macreadie, D. L. McLean, P. G. Thomson et al., "Eyes in the sea: unlocking the mysteries of the ocean using industrial, remotely operated vehicles (ROVs)," Science of the Total Environment, vol. 634, pp. 1077-1091, 2018.

[38] P. A. Di Lillo, E. Simetti, D. De Palma et al., "Advanced ROV autonomy for efficient remote control in the DexROV project," Marine Technology Society Journal, vol. 50, no. 4, pp. 67-80, 2016.

[39] N. R. Sinatra, B. Clark, D. M. Vogt, K. Kit Parker, D. F. Gruber, and R. J. Wood, "Ultragentle manipulation of delicate structures using a soft robotic gripper," Science Robotics, vol. 4, p. 33, 2019.

[40] S. Rúa and R. E. Vásquez, "Development of a low-level control system for the ROV Visor3," International Journal of Navigation and Observation, vol. 2016, Article ID 8029124, 12 pages, 2016.

[41] A. J. Healey, "Model-based maneuvering controls for autonomous underwater vehicles," Journal of Dynamic Systems, Measurement, and Control, vol. 114, no. 4, pp. 614-622, 1992. 
[42] G. Antonelli, "Underwater robots fourth edition," 2018, http://www.springer.com/series/5208.

[43] C. Yang, Y. Wang, and F. Yao, "Driving performance of underwater long-arm hydraulic manipulator system for small autonomous underwater vehicle and its positioning accuracy," International Journal of Advanced Robotic Systems, vol. 14, no. 6, pp. 172988141774710-172988141774718, 2017.

[44] P. Pounds, R. Mahony, and P. Corke, "Modelling and control of a large quadrotor robot," Control Engineering Practice, Elsevier, vol. 18, no. 7, pp. 691-699, 2010.

[45] C. Nicol, C. J. B. MacNab, and A. Ramirez-Serrano, "Robust adaptive control of a quadrotor helicopter," Mechatronics, Elsevier, vol. 21, no. 6, pp. 927-938, 2011.

[46] D. Hanafi, M. Qetkeaw, R. Ghazali, M. N. M. Than, W. M. Utomo, and R. Omar, "Simple GUI wireless controller of quadcopter," International Journal of Communications, Network and System Sciences, vol. 6, no. 1, pp. 52-59, 2013.

[47] M. Orsag, C. M. Korpela, S. Bogdan, and P. Y. Oh, "Hybrid adaptive control for aerial manipulation," Journal of Intelligent \& Robotic Systems, vol. 73, no. 1-4, pp. 693-707, 2014.

[48] Y. Yu, X. Ding, and J. J. Zhu, “Dynamic modeling and control for aerial arm-operating of a multi-propeller multifunction aerial robot," Advanced Robotics, vol. 31, no. 13, pp. 665-679, 2017.

[49] M.-D. Tran and H.-J. Kang, "A novel adaptive finite-time tracking control for robotic manipulators using nonsingular terminal sliding mode and RBF neural networks," International Journal of Precision Engineering and Manufacturing, vol. 17, no. 7, pp. 863-870, 2016.

[50] Y. Wang, F. Yan, J. Chen, F. Ju, and B. Chen, "A new adaptive time-delay control scheme for cable-driven manipulators," IEEE Transactions on Industrial Informatics, vol. 15, no. 6, pp. 3469-3481, 2019.

[51] Y. Wang, L. Liu, D. Wang, F. Ju, and B. Chen, "Time-delay control using a novel nonlinear adaptive law for accurate trajectory tracking of cable-driven robots," IEEE Transactions on Industrial Informatics, vol. 16, no. 8, pp. 5234-5243, 2020.

[52] Y. Wang, F. Yan, J. Chen, and B. Chen, "Continuous nonsingular fast terminal sliding mode control of cable-driven manipulators with super-twisting algorithm," IEEE Access, vol. 6, pp. 49626-49636, 2018.

[53] Z. A. Ali and X. Li, "Controlling of an under-actuated quadrotor UAV equipped with a manipulator," IEEE Access, vol. 8, pp. 34664-34674, 2020.

[54] M. Orsag, C. Michael Korpela, S. Bogdan, and P. Y. Oh, "Hybrid adaptive control for aerial manipulation," Journal of Intelligent \& Robotic Systems, vol. 73, no. 1-4, pp. 693-707, 2014.

[55] M. Ahsan, "Modification of a toy helicopter into a highly cost effective, semi-autonomous, reconnaissance unmanned aerial vehicle," in Proceedings of the 2012 International Conference on Robotics and Artificial Intelligence, ICRAI 2012, pp. 49-54, IEEE, Rawalpindi, Pakistan, October 2012.

[56] U. F. Ahmed, S ur-Rehman, Z. Hameed, F. Ahmed, T. A. U'Chong, and M. M. A. Asif, "Design and development of control system for unmanned ground vehicle and its manipulator," in Proceedings of the 2020 International Conference on Engineering and Emerging Technologies (ICEET), pp. 1-8, Lahore, Pakistan, February 2020.

[57] M. Mustafa, Modeling and Dynamic Control of Autonomous Ground Mobile Manipulators, University of Calgary, Calgary, Canada, 2016.

[58] G. M. Abro, B. Jabeen, Ajodhia et al., "Designing smart sewerbot for the identification of sewer defects and blockages," International Journal of Advanced Computer Science and Applications, vol. 10, no. 2, pp. 615-619, 2019.

[59] R. Jabeen, T. Pathmakumar, T. Venugopal, G. Maruthiyodan, and A. Philip, "DTMF based robotic arm design and control for robotic coconut tree climber," in Proceedings of the 2015 International Conference on Computer, Communication and Control (IC4), Indore, India, September 2015. 